\title{
AUTOMATIC CREATION OF LISTS FROM WIKIPEDIA ARTICLES
}

\author{
Martin Šeleng and Ladislav Hluchý \\ Institute of Informatics, Slovak Academy of Sciences \\ Dúbravská cesta 9, 84507 Bratislava, Slovakia
}

\begin{abstract}
In the paper, we will introduce an approach to automatic list completion based on few keywords specified by the user, using knowledge base constructed previously from English Wikipedia articles. Our approach is similar to Google Sets experiment which does not exist nowadays but its part was integrated in Google Drive (formerly Google Docs) for some time. After writing few words the system recommends additional words to create the list. Our solution is based on offline processing of the English Wikipedia corpus where it searches for tables and lists (as a special case of one column table). These tables/lists are indexed by the system and this index is used to extend user defined keywords by relevant items.
\end{abstract}

\section{KEYWORDS}

Wikipedia, Information Extraction, Automatic Extraction, Completion of Text Lists

\section{INTRODUCTION}

Auto list completion for numbered items, dates, times, and other range-oriented lists is not a special task today. On the other hand, supplementing the lists based on unstructured text is much more difficult and in many cases not fully functioning. One of the first solutions in this area was Google's Google Sets ${ }^{1}$ experiment. This product, after entering few words (the minimum number was 3) supplemented the list with "relevant" items. Unfortunately, Google does not provide API to this product today, but its parts have been integrated into Google Drive ${ }^{23}$.

Our work was partly inspired by Google's product. Google Sets has analysed downloaded pages and searched for structured information such as lists. Concept in our paper seeks to broaden the presented contribution by not only searching the lists but also tables, which in many cases are also useful sources of lists. There are more solutions inspired by Google Sets experiment. Unlike the other ones, our solution does not use the internet to build the lists but one of the largest free downloads - Wikipedia ${ }^{4}$. Other solutions presented on the web mostly work as online tools, searching the web in the time of query, which is really slow.

The paper consists five chapters. In chapter 2, we present existing solutions similar to our framework. Chapter 3 presents the concept of our framework. Chapter 4 contains the evaluation of the results obtained by our solution. In the last chapter we present pros/cons of our approach and possible further research directions.

\footnotetext{
${ }^{1}$ http://labs.google.com/sets - not accessible today

2 http://googlesystem.blogspot.sk/2012/11/google-sets-still-available.html - didn’t work from June 2014

${ }^{3}$ https://drive.google.com

${ }^{4}$ http://www.wikipedia.org/
} 


\section{EXISTING SOLUTIONS}

As mentioned above, most solutions for auto list completion use the internet to extract lists and look for structured information. When designing our solution, we have reviewed existing solutions that can be divided into four groups:

- Creating lists based on page structure

- Google Sets: While Google Sets had never been presented at any conference, the patent filed by Google clearly describes the algorithm used for retrieving the lists. Specifically, Google Sets automatically generates lists of items based on their frequency in lists on the internet. Google Sets automatically extracts information from HTML tags: $\langle\mathbf{U L}\rangle,\langle\mathbf{O L}\rangle,\langle\mathrm{DL}\rangle$, and $\langle\mathbf{H 1}\rangle \ldots . \mathbf{H 6}\rangle$.

- WebTables [5]: WebTables authors worked or are still working at Google, so their solution is similar to Google Sets. But unlike Google Sets, they extract lists from specific tables (HTML tag <TABLE >) while trying to understand the scheme and integrate all data from these tables in one list.

- Mining Data Records [6]: The Mining Data Records framework seeks to get lists by analysing the DOM structure of each website. This solution is based on two observations:

1. A list of data records (item list) is in a coherent web page area and are similarly formatted (using similar HTML tags and patterns)

2. Records in the data area are assigned to one parent node. Consequently, the probability that HTML $\langle\mathbf{U L}\rangle$ and $\langle$ DIV $\rangle$ tags will separate lists is approximately the same.

- Creating online wrappers to get lists

- RoadRunner [7]: The RoadRunner algorithm is able to understand the structure of a website and create an online wrapper for this site. The resulting wrapper is very effective in extracting structured repeating objects, but cannot be modified to extract lists from the web in general.

- Set Expander for Any Language [8]: The algorithm used in this solution is similar to the RoadRunner algorithm - it creates a wrapper for any web page. However, unlike the RoadRunner solution, this wrapper is created at the time of search query, but needs multiple keywords to complete the set. In addition, since the wrapper is formed at the time of demand, it is extremely slow ${ }^{6}$.

- Extracting lists based on visual object layout

- Visual List Extraction [1]: The Visual List Extraction algorithm represents the generated web page as an array of areas and therefore attaches great importance to the visual appearance of the page. The area has a given position and size. The area may include: text, images, or other areas. Each area is actually represented by a DOM object. So all objects that are defined within a region (siblings) are considered part of the list.

- Using Wikipedia articles to extract lists

- Word Grab Bag ${ }^{7}$ : Only few useful information about Word Grab Bag solution can be found. One of them says that the solution is mainly based on word $2 \mathrm{vec}^{8}$ framework developed by Google [9]. It processes Wikipedia articles and searches for similar words based on the relative distance between them.

\footnotetext{
${ }^{5}$ http://www.w3.org/DOM/

${ }^{6} \mathrm{http} / / / \mathrm{www}$. boowa.com/ - not accessible anymore, redirected to the original author webpage

${ }^{7}$ http://wordgrabbag.com/

${ }^{8}$ https://code.google.com/archive/p/word2vec/
} 


\section{WIKIPEDIA SETS EXTRACTOR}

In our solution, we focused on extracting tables and lists from Wikipedia pages, so we focused on offline processing of Wikipedia articles. Our solution does not depend on the amount of information downloaded from the web, but on the free download of Wikipedia. There were 5,871,771 ${ }^{9}$ articles (without redirects, talks, users and any other special pages) in English Wikipedia at the time of writing. This version has packed size 15GB (67GB extracted). Since this is a relatively large amount of data, we decided to process it on clusters with 14 computers, each with 24 cores (HTT enabled). Wikipedia can be downloaded in XML, or as an SQL database export. For our distributed processing, the XML version is much more practical. We used the Apache Hadoop ${ }^{10}$ framework (especially Apache Spark ${ }^{11}$ ) for distributed processing. Since Hadoop can only work with two types of data by default, a sequential file (native format) and a text file, we had to implement support for parsing XML ${ }^{12}$ MediaWiki $^{13}$ format over which Wikipedia is built. Another problem was the extraction of tables and lists because the parser ${ }^{14}$ used in MediaWiki is quite robust and allows tables and lists that do not fully meet the specification. We tested several solutions in Scala or Java ${ }^{1516}$, but none of them suited us. After these experiences we switched to Python and used the WikiTextParser ${ }^{17}$ combined with our own parser, based on regular expressions. The following figure shows the table in MediaWiki format.

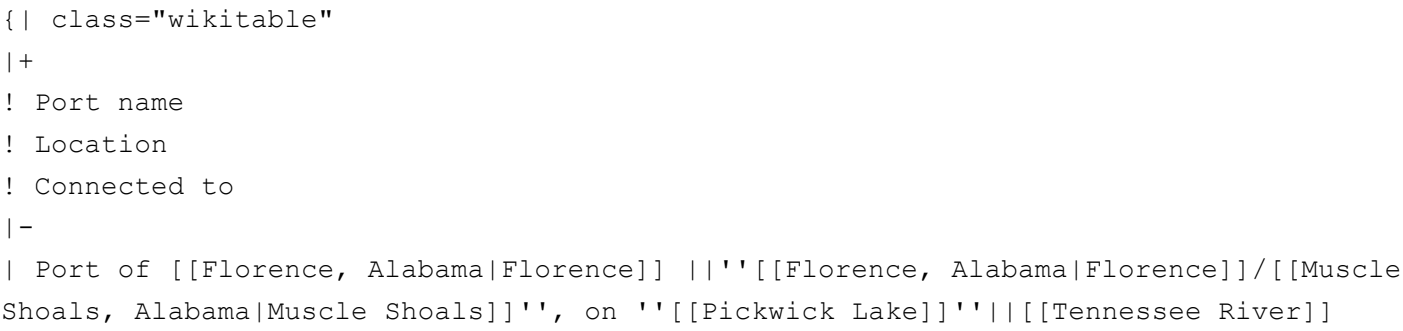

Figure 1. Snippet of the table raw data in MediaWiki format below.

After processing the raw data and removing the line editing formatting, we get the final table as shown

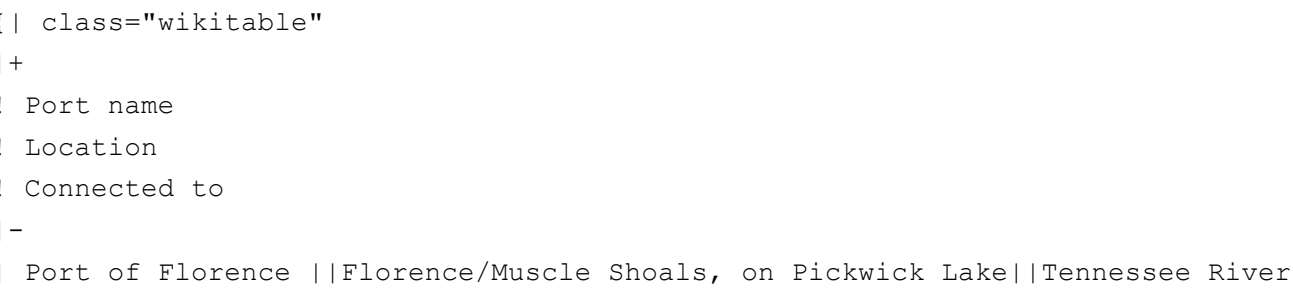

Figure 2. Table processed by our system

\footnotetext{
${ }^{9}$ https://en.wikipedia.org/wiki/Wikipedia:Database_reports/Page_count_by_namespace

${ }^{10} \mathrm{http}$ //hadoop.apache.org/

${ }^{11} \mathrm{https}$ ///spark.apache.org/

12 https://github.com/databricks/spark-xml

$13 \mathrm{http} / / /$ www.mediawiki.org/wiki/MediaWiki

${ }^{14}$ https://github.com/wikimedia/parsoid

$15 \mathrm{http} / / /$ wiki.eclipse.org/Mylyn/Incubator/WikiText

16 http://wikipedia-miner.cms.waikato.ac.nz/

${ }^{17}$ https://github.com/5j9/wikitextparser\#wikitextparser
} 
It is similar with the lists that have a relatively simpler structure than tables:

Table 1. Different list types used in MediaWiki format

\begin{tabular}{ll}
\hline Item & Type \\
\hline$*$ & bulleted list \\
$\#$ & numbered list \\
$;,:$ & term and definition \\
\hline
\end{tabular}

* [ [Category:American male film actors/List of American film actors]]

*[[Category:American film actresses/List of American film actresses]]

*[[Category:American male television actors/List of American television actors]]

* [ [Category:American television actresses|List of American television actresses]]

Figure 3. List raw data in MediaWiki format

However, lists and tables may be arbitrarily combined which may cause confusion in the parsing. Each table column is extracted and creates an individual list. Then, if we want to combine several columns together to create larger lists in the future, we can achieve it easily as the ID of each column is created by the title of a parsed page, hash of the whole table and hash of the column (e. g. 'List of awards and nominations received by Alizée -5206743334941503777 6310303203805118876'). We processed 14,583,057 articles (it is much more than the number in previous paragraph, but the dump consisted of all types of Wikipedia articles) in 17 minutes, but it needed to be executed only once. We extracted 2,482,517 tables (containing 12,903,675 columns/lists) and 18,548,254 lists from the Wikipedia dump (after removing empty and duplicate lists our knowledge base contained 31,141,718 lists). Completely stored datasets are 2.7G ID hashes (the long hash presented before is hashed once again to keep the index smaller) and 8.1G individual lists ([KEY, VALUE] pairs [ID, KEYWORD]). The search function works over these datasets by specifying one or more desired keyword(s). It was executed again on the Apache Spark as we did not want to load whole index into memory. The search took around 40 seconds to return the list extended by keywords suggested by the system. As the keywords entered by the users can be found in several lists, we need to specify ranking function to merge these lists and order them somehow. We found an inspiration in classic tf-idf (term frequency - inverse document frequency) formula:

$$
t f i d f(t, d, D)=t f(t, d) * i d f(t, D)
$$

Equation 1. General tf-idf equation

Of course, we can compute $t f$ and $i d f$ using many other formulas. In the paper we present only those needed for our computation:

$$
t f(t, d)=\frac{f_{t, d}}{\sum_{t, \in d} f_{t, d}}
$$

Equation 2. Term frequency adjusted for all keywords in document

Equation 2 presents the classical relative frequency formula, but in our case the numerator is always 1 as we remove duplicate keywords from columns. As every keyword is in the list only once (we have removed the duplicates inside lists) in the denominator we can simply take the length of the list/column.

$$
t f(t, d)=\frac{1}{|d|}
$$

Equation 3. Term frequency adjusted for list length

We will not present the idf formula, because we do not count on idf. In idf computation, the keyword is penalized if it occurs in many documents (lists). We only use $d f$ (document frequency) formula:

$$
d f(t, D)=\frac{|d \in D: t \in d|}{|D|}
$$

Equation 4. Document frequency adjusted for corpus length 
After these partial equations, we can simply compute $t f d f$, which we will use as a ranking algorithm for adding keywords in user pre-defined lists:

$$
t f d f(t, d, D)=t f(t, d) * d f(t, D)
$$

Equation 5. Document frequency combined with term frequency

\section{RESULTS}

In this chapter, we present some of the results we obtained by our system. It is hard to evaluate such results. The only way is to use humans to decide what should be part of the list and what should not. We tried to prepare several queries (ten queries, with some modifications to main query) with human build answers (looking into Wikipedia or other internet sources) and we compared (where applicable) system results to manually built lists.

By using the first query, we wanted to obtain the list of American presidents, using the keywords "Ronald Reagan" and "Jimmy Carter". The query resulted in the list ${ }^{18}$ shown in Table 1.

On the $17^{\text {th }}$ position there is a result "President"19, with score of 1.49 . On the $27^{\text {th }}$ position there is a result "List of Presidents of the United States", with score of 0.57. If we add additional keyword "Donald Trump" to the list, only the scores changed and "John F. Kennedy" will replace "Gerald Ford" in top ten results. Result "President" moves to the $19^{\text {th }}$ position and the "List of Presidents of the United States" leaves top 30 results.

Table 1. List of American presidents

\begin{tabular}{lr}
\hline Keyword & Score \\
\hline Ronald Reagan & 26.39 \\
Jimmy Carter & 26.39 \\
Bill Clinton & 17.87 \\
George W. Bush & 17.04 \\
Barack Obama & 16.68 \\
George H. W. Bush & 13.32 \\
Richard Nixon & 11.05 \\
Lyndon B. Johnson & 7.75 \\
Donald Trump & 7.47 \\
Gerald Ford & 6.05
\end{tabular}

Table 2. List of humans walked on the Moon

\begin{tabular}{lr}
\hline Keyword & Score \\
\hline Alan Shepard & 0.12 \\
Ed Mitchell & 0.12 \\
Neil Armstrong & 0.12 \\
Gene Cernan & 0.12 \\
Jim Irwin & 0.12 \\
Alan Bean & 0.12 \\
Astronaut & 0.12 \\
John Young & 0.12 \\
Pete Conrad & 0.12 \\
Janice Voss & 0.01
\end{tabular}

The next list we want to complete is the list of astronauts who walked on the Moon, by adding two initial keywords "Neil Armstrong" and "Gene Cernan". The results are shown in Table 2. Now we can see that the $10^{\text {th }}$ result is completely wrong and that there are few astronauts missing. Looking at the score, we can see that the result "Astronaut" is probably from the header, "Janice Voss" comes from another table/list. Other nine results are probably all from one table/list (regarding the scores). Following results are the names of Russian cosmonauts, with the same score as the $10^{\text {th }}$ result (which is completely wrong). Looking into Wikipedia webpage, we can actually find that there is an article "List of people who have walked on the Moon" 20 , which contains all 12 astronaut names walking on the Moon and our system should found the results. Closer look into the article reveals that Wikipedia templates ${ }^{21}$ are used inside the table and our system can't process templates yet, because of many different templates and parsing difficulties.

Other query to the system was about returning car manufacturers with specifying keywords "Kia", "BMW", "Honda". The results are shown in Table 3. The results are promising in this case, too, with problems only on the $17^{\text {th }}$ and the $26^{\text {th }}$ position, with "Manufacturer" and "Constructor" results respectively. These results are probably headers from the tables used in this list. If we add keywords "Volvo", "Citroën", "Audi" to the query, the results are meshed a little bit ("Volvo", "Citroën", "Audi" are inside top 10) and scores are lowered little bit (top result has score of 0.13 ). But there is no incorrect answer in top 30 results.

\footnotetext{
${ }^{18}$ All lists will be limited at 10 keywords

${ }^{19} \mathrm{We}$ are not removing first row from the table as lot of tables do not have headers

${ }^{20} \mathrm{https}$ ///en.wikipedia.org/wiki/List_of_people_who_have_walked_on_the_Moon

${ }^{21} \mathrm{https}$ ://en.wikipedia.org/wiki/Wikipedia:Templates
} 
Now let us say we want to fill the list of France regions, specifying four of them: "Burgundy", "Aquitaine", "Limousin", "Alsace" (Table 4.). This is a kind of tricky question as France changed the names of regions in 2015-2016, grouping some of them together under new names. However, all the keywords used in the query were old names so the answers were correct. When we use a query with two new region names "Nouvelle-Aquitaine" and "Grand Est", the results (shown in Table 5) contain new names and all of them are correct, except the result on the $13^{\text {th }}$ position, where a header keyword ("Region") is again. The results below the $13^{\text {th }}$ position contain also overseas territories of France.

Table 3. Car manufacturers

\begin{tabular}{lr}
\hline Keyword & Score \\
\hline Honda & 1.26 \\
BMW & 1.26 \\
Kia & 1.06 \\
Mazda & 0.75 \\
Ford & 0.59 \\
Toyota & 0.51 \\
Nissan & 0.48 \\
Volkswagen & 0.45 \\
Hyundai & 0.35 \\
Chevrolet & 0.33
\end{tabular}

Table 4. France political regions

\begin{tabular}{lr}
\hline Keyword & Score \\
\hline Alsace & 0.75 \\
Aquitaine & 0.75 \\
Auvergne & 0.75 \\
Burgundy & 0.75 \\
Limousin & 0.75 \\
Lorraine & 0.75 \\
Picardy & 0.75 \\
Franche-Comté & 0.67 \\
Poitou-Charentes & 0.64 \\
Champagne-Ardenne & 0.64
\end{tabular}

The next query is on (active) German football players, specifying three of them "Toni Kroos", "Sami Khedira" and "Thomas Müller". Looking at the results in Table 6, we can see that the $5^{\text {th }}$ result is obviously an error, as "Joachim Löw" is a former football player but today he is a coach of German national team. Also the results "Manager:" and "Substitutions:" are wrong. However, results from the $10^{\text {th }}$ to the $30^{\text {th }}$ position are correct as each listed player comes from Germany.

Table 5. New France political regions

\begin{tabular}{lr}
\hline Keyword & Score \\
\hline Grand Est & 2.11 \\
Hauts-de-France & 2.11 \\
Nouvelle-Aquitaine & 2.11 \\
Auvergne-Rhône-Alpes & 1.94 \\
Occitanie & 1.86 \\
Bourgogne-Franche-Comté & 1.74 \\
Normandy & 1.50 \\
Pays de la Loire & 1.41 \\
Centre-Val de Loire & 1.29 \\
Provence-Alpes-Côte d'Azur & 1.10
\end{tabular}

Table 6. German football players

\begin{tabular}{lr}
\hline Keyword & Score \\
\hline Toni Kroos & 2.12 \\
Sami Khedira & 2.12 \\
Thomas Müller & 2.12 \\
Mesut Özil & 2.06 \\
Joachim Löw & 2.01 \\
Jérôme Boateng & 1.90 \\
Manager: & 1.90 \\
Bastian Schweinsteiger & 1.30 \\
Substitutions: & 0.98 \\
Benedikt Höwedes & 0.93
\end{tabular}

The query to complete the list of European countries, containing three keywords "Slovakia", "Italy", "Germany", ends with the results shown in Table 7. The first mistake is on the $30^{\text {th }}$ position ("Country", with score of 5.76), and from the $35^{\text {th }}$ position on there are only few European countries left - $36^{\text {th } ~ " M a l t a ", ~} 41^{\text {st }}$ "Belarus", followed by several former republics of Yugoslavia.

Table 7. European countries list

\begin{tabular}{ll}
\hline Keyword & Score \\
\hline Italy & 28.91 \\
Germany & 28.91 \\
Slovakia & 28.84 \\
France & 23.17 \\
Spain & 20.31 \\
Sweden & 16.82 \\
Poland & 16.74 \\
Netherlands & 15.69 \\
Austria & 14.80 \\
Czech Republic & 14.76
\end{tabular}

Table 8. Athletic sports

\begin{tabular}{lr}
\hline Keyword & Score \\
\hline Javelin & 17.04 \\
Shot put & 12.66 \\
Long jump & 12.57 \\
Discus & 11.08 \\
High jump & 6.70 \\
Event & 5.16 \\
Pole vault & 4.31 \\
Triple jump & 3.83 \\
$100 \mathrm{~m}$ & 3.19 \\
$400 \mathrm{~m}$ & 3.07
\end{tabular}


If we want to get a list of athletic sports, we can specify a query to our system with keywords "javelin", "long jump", "shot put". The results can be seen in Table 8. Only result "Event" is a mistake (probably again because of a header issue). In top 40 results, all the listed items are correct, except we have again keywords as "Long Jump", "High Jump" (capital letters issue), etc., which should be merged with the results already added to the list.

When trying to retrieve European capital cities (Table 9.) by initialling the search over six keywords: "Bratislava", "Prague", "Vienna", "Budapest", "Berlin", "Paris" (we used so many as an input because we didn't want to retrieve other major European cities only capital ones), we receive the results with the first error at the $25^{\text {th }}$ position ("Milan"). Below the $25^{\text {th }}$ position, there is a mix of good and bad answers.

The last predefined query is about receiving the list of Himalayan peaks with keywords "Mount Everest", "K2", "Nanga Parbat". The results can be seen in Table 10. These results are correct, missing only on the $15^{\text {th }}$ and $16^{\text {th }}$ positions with results "Mountain" and "Peak" (header issue).

Table 9. European capital cities

\begin{tabular}{lr}
\hline Keyword & Score \\
\hline Bratislava & 0.87 \\
Berlin & 0.87 \\
Vienna & 0.87 \\
Budapest & 0.87 \\
Paris & 0.87 \\
Prague & 0.87 \\
London & 0.77 \\
Stockholm & 0.77 \\
Copenhagen & 0.77 \\
Helsinki & 0.67
\end{tabular}

Table 10. Himalayan peaks

\begin{tabular}{lr}
\hline Keyword & Score \\
\hline Mount Everest & 0.34 \\
Dhaulagiri & 0.34 \\
Manaslu & 0.34 \\
Nanga Parbat & 0.34 \\
K2 & 0.34 \\
Makalu & 0.28 \\
Broad Peak & 0.28 \\
Lhotse & 0.28 \\
Gasherbrum II & 0.28 \\
Cho Oyu & 0.28
\end{tabular}

\section{CONCLUSION}

The first results of our system, described in chapter 4, evaluated manually by viewing the extracted data, show that the selected approach is suitable for extracting and extending user-specified lists. It is not an easy task to evaluate our solution, but our next goal will be to design a semi-automatic method for evaluating our system and comparing it with existing solutions. The problems which arise in the evaluation of our system can be divided into categories:

- technical:

o build more robust parser (mainly for templates parsing),

o problems with capital letters (e.g. "Ice hockey" and "Ice Hockey" in Table 8.); it is not such a big problem, but if we do it probably we can combine things, that shouldn't be merged together,

o each list created by our system has at least few dozen results; we need to specify the function which will drop rest of the results based on the decreasing score in the list and length of the list,

o problem with the time used by search; should be lowered to milliseconds and not seconds

- $\quad$ semantical/logical:

o some items use different names in the tables/lists, e.g. "Edgar Mitchell" is referred also as "Ed Mitchel" (Table 2.),

o trying to solve the header problems in the tables by some probability function, which will remove common keywords as "Name", "Manager", etc.

o using only subset of input keywords (it will be enough that column contains only part of the input keywords and not all of them) and merging lists together, how-to process sub-lists from Wikipedia articles (e.g. lowering the priority, ...). 


\section{ACKNOWLEDGEMENT}

This work is being developed with the support of projects: APVV-17-0619 and VEGA 2/0167/16.

\section{REFERENCES}

B. Liu, R. Grossman, and Y. Zhai. Mining data records in web pages. In KDD, pages 601-606, New York, NY, USA, 2003. ACM

Cafarella M. J. et al, 2008, Webtables: exploring the power of tables on the web. PVLDB, pages 538-549

Crescenzi V. et al, 2002, Roadrunner: automatic data extraction from data-intensive web sites. In SIG-MOD, pages 624-624, New York, NY, USA, 2002. ACM

Mikolov T. et al, 2006, Distributed Representations of Words and Phrases and their Compositionality, Advances in Neural Information Processing Systems 26, https://papers.nips.cc/paper/5021-distributed-representations-of-words-andphrases-and-their-compositionality.pdf

Wang R. C. et al, 2008, Automatic Set Expansion for List Question Answering, EMNLP '08 Proceedings of the Conference on Empirical Methods in Natural Language Processing Pages 947-954

Wang R. C., Cohen W. W., 2007 Language-independent set expansion of named entities using the web. In ICDM

Wang R. C., Cohen W. W., 2009. Automatic Set Instance Extraction using the Web, ACL '09 Proceedings of the Joint Conference of the 47th Annual Meeting of the ACL and the $4^{\text {th }}$ International Joint Conference on Natural Language Processing of the AFNLP: Volume 1 Pages 441-449

Wang R. C., Cohen W. W., 2009. Character-level analysis of semi-structured documents for set expansion, EMNLP '09 Proceedings of the 2009 Conference on Empirical Methods in Natural Language Processing: Volume 3 Pages 1503-1512

Weninger T. et al, December 2010. Unexpected Results in Automatic List Extraction on the Web. ACM SIGKDD Explorations Newsletter archive, Volume 12 Issue 2, Pages 26-30 